\title{
Case Report: Anaplastic Astrocytoma Treated with Postoperative Radiotherapy Using Flattening Filter-Free Volumetric Arc Therapy (FFF-VMAT) during Pregnancy-Acceptable Fetal Dosimetry
}

\author{
Tomoyuki Noyama, Wataru Takahashi, Yuki Nozawa, Kanabu Nawa, Hideomi Yamashita*, \\ Osamu Abe, Keiichi Nakagawa
}

Department of Radiology, The University of Tokyo Hospital, Bunkyo-ku, Tokyo, Japan

Email: *yamachan07291973@yahoo.co.jp

How to cite this paper: Noyama, T., Takahashi, W., Nozawa, Y., Nawa, K., Yamashita, H., Abe, O. and Nakagawa, K. (2020) Case Report: Anaplastic Astrocytoma Treated with Postoperative Radiotherapy Using Flattening Filter-Free Volumetric Arc Therapy (FFF-VMAT) during Pregnancy-Acceptable Fetal Dosimetry. Journal of Cancer Therapy, 11, 738-744. https://doi.org/10.4236/jct.2020.1111063

Received: October 30, 2020

Accepted: November 23, 2020

Published: November 26, 2020

Copyright $\odot 2020$ by author(s) and Scientific Research Publishing Inc. This work is licensed under the Creative Commons Attribution International License (CC BY 4.0).

http://creativecommons.org/licenses/by/4.0/

(c) (i) Open Access

\begin{abstract}
Background: Postoperative irradiation for brain tumor in pregnant women is a matter of concern. Aim: We aimed to assess the safety of radiotherapy for brain tumors in pregnancy. We here report a successful treatment for anaplastic astrocytoma during pregnancy: surgery + postoperative irradiation. We wish to emphasize how we devised irradiation procedure to achieve both therapeutic effectiveness and safety to the fetus/infant. Case Presentation: A 34-year-old pregnant woman suffered with brain anaplastic astrocytoma. Tumor resection under craniotomy was performed with success. We decided to conduct postoperative radiotherapy at 25 weeks of gestation to reduce the risk of recurrence. We used a flattening filter-free volumetric arc therapy (FFF-VMAT) technique, which can achieve lower out-of-field dose than VMAT with a flattening filter or helical tomotherapy. We prescribed $60 \mathrm{~Gy}$ over 30 fractions. During actual beam delivery, surface and rectal dose to the patient (mother) were measured. The total fetal dose was estimated at 0.006 $0.018 \mathrm{~Gy}$, which is under the threshold set by the ICRP. A male healthy infant was born vaginally at the 37th week of pregnancy. The patient (mother) and the infant are healthy at the time of writing. Conclusion: FFF-VMAT is a good choice for brain tumors during pregnancy.
\end{abstract}

\section{Keywords}

Pregnancy, Radiotherapy, Flattening Filter-Free, Volumetric Arc Therapy, Anaplastic Astrocytoma, Fetal Dose 


\section{Introduction}

Brain tumors in pregnancy are uncommon. Anaplastic astrocytoma (AA; World Health Organization [WHO] grade III) is a diffusely infiltrative astrocytic brain tumor with anaplasia and represents $3.3 \%$ of primary brain tumors, with a 5 -year overall survival rate of $44.3 \%$ when treated with surgery and chemoradiotherapy [1].

Resection and adjuvant radiotherapy are recommended for nongravid patients with AA [2]. Because of fetal radiosensitivity, radiotherapy for pregnant patients is generally avoided. However, there are cases where delayed treatment would increase the risk of morbidity and mortality, necessitating treatment during pregnancy. Here, we report a case of AA treated with postoperative FFF-VMAT during pregnancy.

\section{Case Report}

A 34-year-old woman, gravida 2, para 1, was admitted to our hospital at 14 weeks of gestation because of sudden convulsions. Brain magnetic resonance imaging (MRI) detected a $4 \mathrm{~cm}$ region of edema on T2-weighted images (T2WI) with a small enhancing nodule in the right frontal lobe on enhanced T1-weighted images (T1WI) (Figure 1). Because of the likelihood of AA, craniotomy before delivery was desirable.

Craniotomy and tumor resection were performed at 20 weeks. Gross total resection of the tumor and a small margin was achieved with no complications. Pathology confirmed grade III AA with the isocitrate dehydrogenase (IDH) mutation (Figure 2).

A multidisciplinary meeting concluded that postoperative radiotherapy would be conducted at 25 weeks of gestation to reduce the risk of local failure and to decrease the risk of fetal developmental delay, and that induction of labor or cesarean section would be performed at 37 weeks. The patient would then receive adjuvant chemotherapy with procarbazine, nimustine, and vincristine.
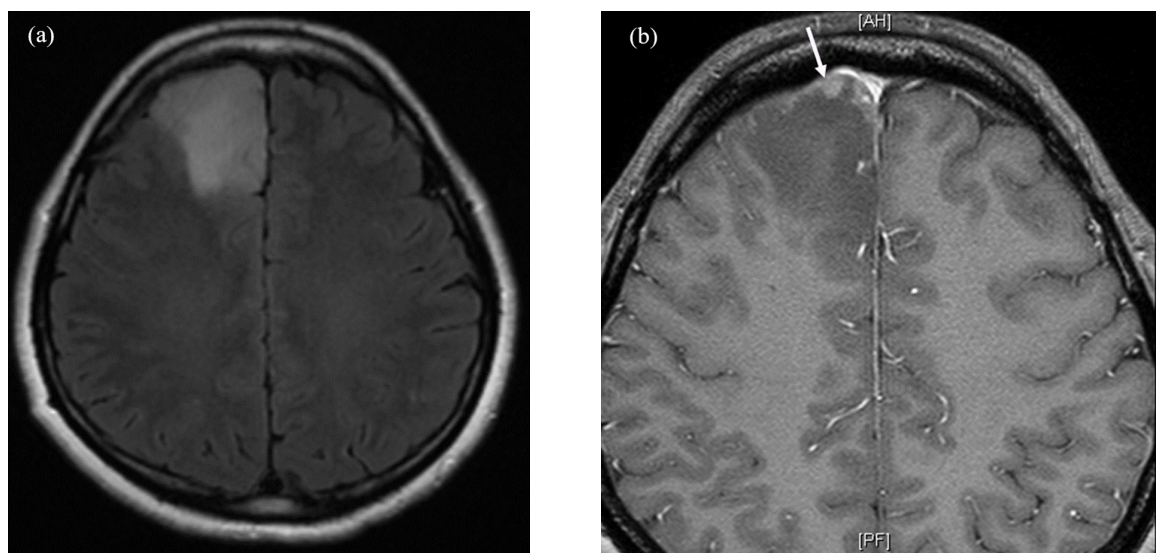

Figure 1. Preoperative T2-weighted (a) and gadolinium-enhanced T1-weighted (b) brain magnetic resonance imaging (MRI) scan. $4 \mathrm{~cm}$ edematous region in the right frontal lobe is present in T2WI and an enhancing nodule (white arrow) is seen on the T1WI. 

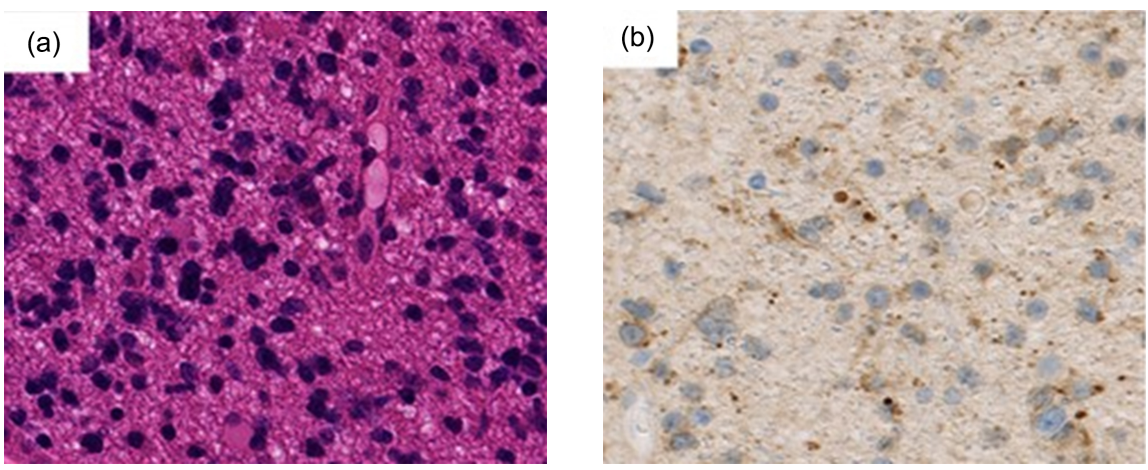

Figure 2. Pathology of the brain tumor. (a) HE staining. (b) Immunohistological staining of mutant isocitrate dehydrogenase 1 (mIDH-1).

The planning CT was carried out using a 16-detector CT scanner (Toshiba Aquilion LB, Toshiba Medical Systems, Otawara, Japan) and reconstructed as 2 mm sections. FFF-VMAT was selected because of its association with decreased photon scatter from the flattening filter and lower out-of-field exposure compared to VMAT with a flattening filter and helical tomotherapy [3] [4] [5]. The planning CT data were imported into our treatment planning system and fused with the preoperative and postoperative MRI data.

The clinical target volume (CTV) included the preoperative and postoperative edema plus a $1.5 \mathrm{~cm}$ margin (CTV1) and the tumor bed plus a $1.5 \mathrm{~cm}$ margin (CTV2), both adjusted for anatomical barriers. The planning target volume (PTV) included the CTV plus a $0.5 \mathrm{~cm}$ margin. We prescribed 50 Gy to PTV1 and 60 Gy to PTV2 over 30 fractions for 6 weeks using a simultaneous integrated boost method. The treatment planning was performed with Pinnacle (v9.10, Philips Medical Systems, Fitchburg WI, USA) with $6 \mathrm{MV}$ photons and a maximum dose rate of $1500 \mathrm{MU} / \mathrm{min}$ for a Synergy linear accelerator with an Agility multileaf collimator (Elekta AB, Stockholm, Sweden). The FFF-VMAT plans were performed with one complete arc (Figure 3). The dose constraints for the PTVs were as follows: median values of $\mathrm{D} 2 \%, \mathrm{D} 10 \%$, and $\mathrm{D} 50 \%$ were $<120 \%, 110 \%$, and $105 \%$ of the prescribed dose, respectively. The PTV coverage of the plan was $95 \%$. Dose constraints for the mean dose to the lenses were $<6 \mathrm{~Gy}$. Dose constraints for the maximum dose to the orbits, optic nerves, chiasm, and brainstem were $<40 \mathrm{~Gy},<50 \mathrm{~Gy},<50 \mathrm{~Gy}$, and $<54 \mathrm{~Gy}$, respectively.

Past reports have suggested decreasing fetal dose using lead shielding [6] [7]. However, we did not use any shielding devices in this treatment because there were safety concerns, such as the risk of it collapsing by its own weight.

The fetal dose was first estimated using a phantom. To measure surface dose, glass dosimeters were placed in the midline between a point $29 \mathrm{~cm}$ caudal to the superior margin of the manubrium sterni and the pubic symphysis. Glass dosimeters were also placed inside the phantom to measure internal dose.

The dose to the phantom from one fraction is presented in Figure 4 . The estimated total fetal dose would be 0.009 - 0.024 Gy, lower than the 0.1 Gy cutoff point of the ICRP [8]. 

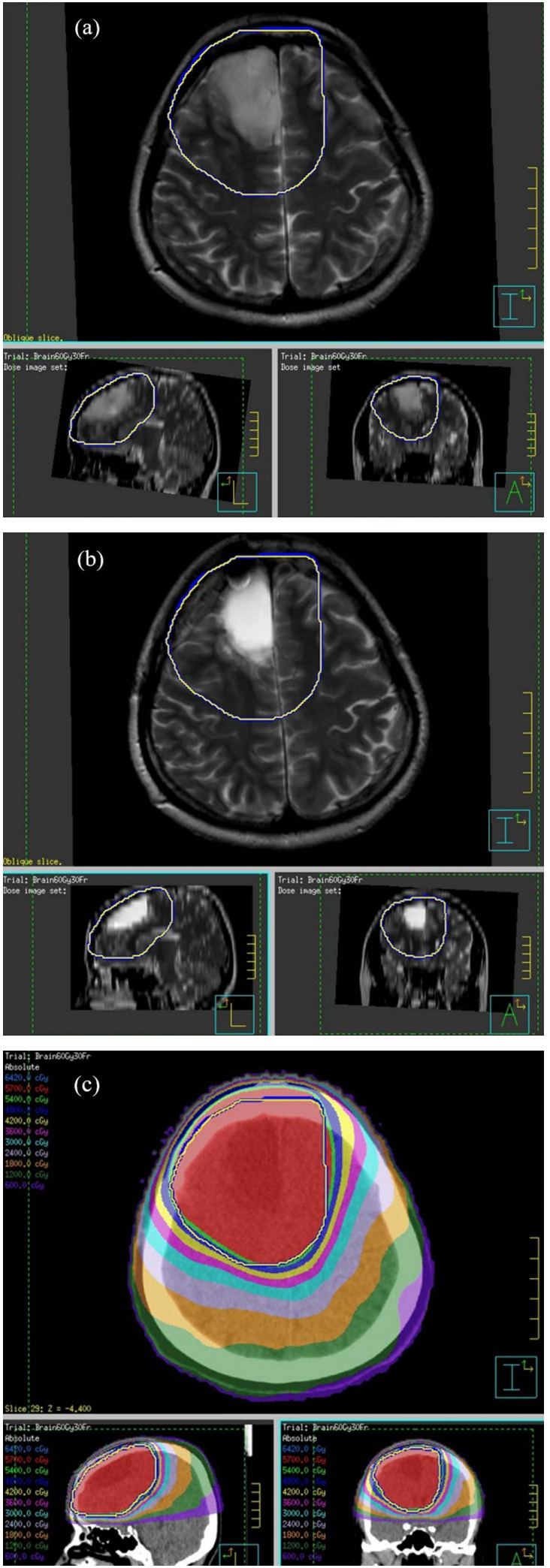

Figure 3. Treatment plans for flattening filter-free VMAT (FFF-VMAT). (a) A preoperative T2WI. (b) A postoperative T2WI. The yellow and blue lines demarcate PTV1 and PTV2, respectively. These PTVs include the edema. (c) The red color wash illustrates the 95\% (57 Gy) isodose. 


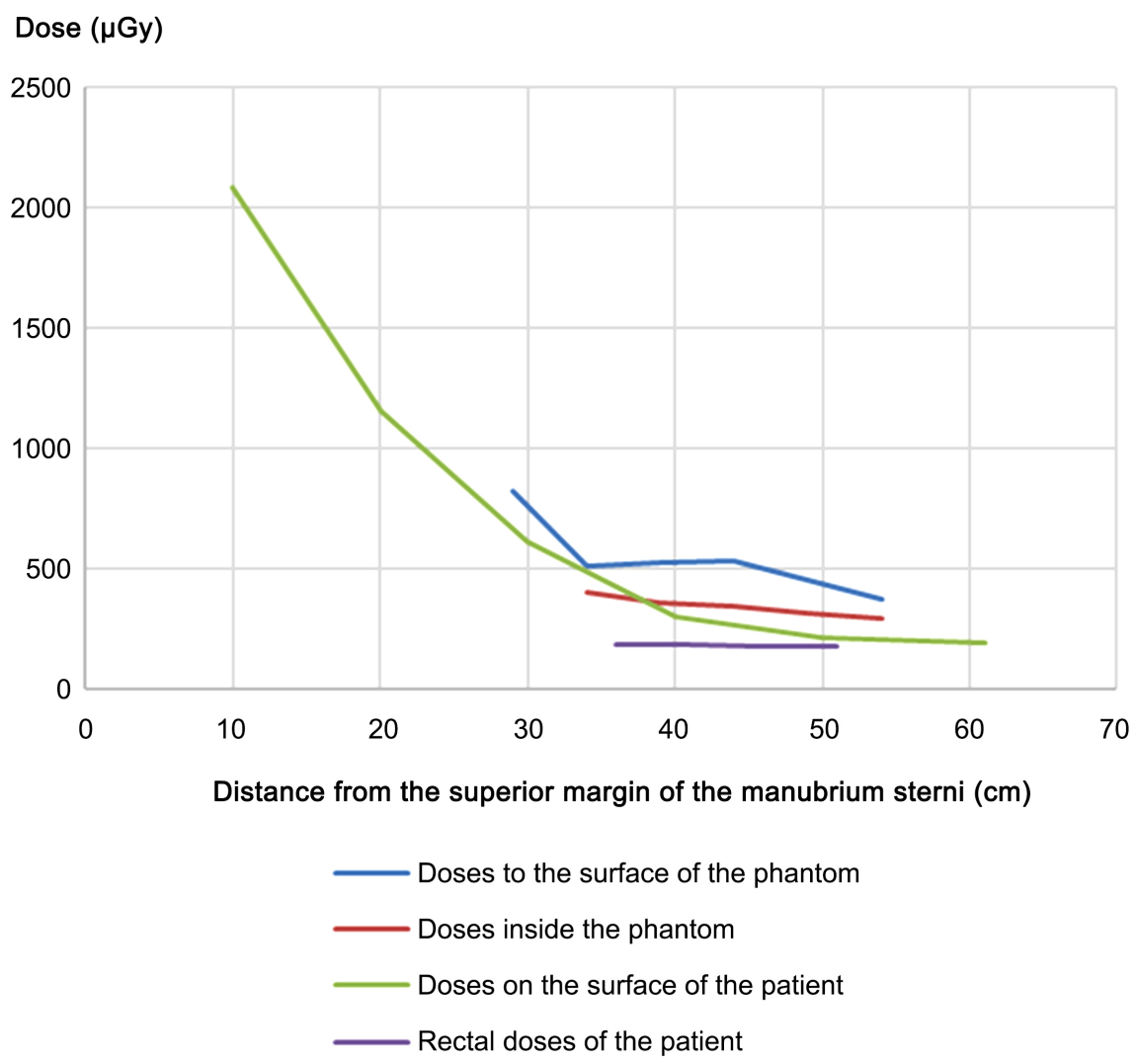

Figure 4. Out-of-field dose of one fraction for flattening filter-free VMAT (FFF-VMAT) vs. distance from the superior margin of the manubrium sterni of the patient/phantom.

During beam delivery, surface doses were measured using six glass dosimeters starting $10 \mathrm{~cm}$ caudal to the superior margin of the manubrium sterni and extending to the pubic symphysis. Rectal doses were measured using four glass dosimeters placed between 10 and $25 \mathrm{~cm}$ from the anal verge.

The measured total fetal dose was $0.006-0.018$ Gy (Figure 4), less than the threshold dose for deterministic effects such as fetal developmental delay [9]. The radiotherapy was completed as scheduled. Grade 2 alopecia was the only adverse event.

Labor was induced at 37 weeks and a healthy boy was born vaginally. He had no complications at the last follow-up.

\section{Discussion}

Radiotherapy for pregnant patients should be avoided, but often there is no alternative. In the first trimester, pregnancy termination followed by radiotherapy is sometimes conducted. If the patient is in the second or third trimester, the risk to the fetus is thought to be relatively low and conducting radiotherapy without termination is considered. Exposure to radiation can cause malformation from 2 to 8 weeks and can result in developmental delay from 8 to 25 weeks of gestation. The risk of developmental delay decreases rapidly after 25 weeks and therefore radiotherapy was conducted at 25 weeks. We were able to achieve an 
acceptable fetal dose, which was under the threshold dose for deterministic effects [9].

Past reports have observed that FFF-VMAT achieves a lower fetal dose than VMAT with a flattening filter [3]. In helical tomotherapy, mainly due to the large number of MUs, higher fetal doses resulted than those measured with VMAT with a flattening filter and FFF-VMAT [4]. Therefore, we selected FFF-VMAT to treat the patient.

We did not attempt 3-dimensional conformal radiotherapy (3D-CRT) in our case, though IMRT has scatter and leakage issues compared to 3D-CRT [10]. We hoped to use IMRT to decrease the risk of adverse effects, especially brain necrosis. We observed an acceptable fetal dose, similar to those in previous cases of brain tumors in pregnancy treated by 3D-CRT or fixed-beam IMRT [11] [12] [13] [14], in which a flattening filter was used. The observed dose to the patient was lower than that to the phantom (Figure 4), probably because of the differences in the shape and composition between the patient and the phantom.

\section{Conclusion}

FFF-VMAT is a suitable choice for brain tumors in pregnancy.

\section{Acknowledgements}

We thank Libby Cone, MD, MA, from DMC Corp. (http://www.dmed.co.jp; http://www.dmed.co.jp/) for editing drafts of this manuscript.

\section{Consent for Publication}

Written informed consent was obtained from the patient for the publication of this case report.

\section{Conflicts of Interest}

The authors declare no conflicts of interest regarding the publication of this paper.

\section{References}

[1] Committee of Brain Tumor Registry of Japan (2017) Report of Brain Tumor Registry of Japan (2005-2008) 14th Edition. Neurologia Medico-Chirurgica (Tokyo), 57, 9-102.

[2] Walker, M.D., et al. (1978) Evaluation of BCNU and/or Radiotherapy in the Treatment of Anaplastic Gliomas. A Cooperative Clinical Trial. Journal of Neurosurgery, 49, 333-343. https://doi.org/10.3171/jns.1978.49.3.0333

[3] Alvarez Moret, J., et al. (2018) Second Cancer Risk after Radiation Therapy of Ependymoma Using the Flattening Filter Free Irradiation Mode of a Linear Accelerator. Journal of Applied Clinical Medical Physics, 19, 632-639. https://doi.org/10.1002/acm2.12438

[4] Takahashi, W., et al. (2019) Acceptable Fetal Dose Using Flattening Filter-Free Volumetric Arc Therapy (FFF VMAT) in Postoperative Chemoradiotherapy of Tongue Cancer during Pregnancy. Clinical and Translational Radiation Oncology, 20, 9-12. https://doi.org/10.1016/j.ctro.2019.10.002 
[5] Kim, D.W., et al. (2014) Risk of Secondary Cancers from Scattered Radiation during Intensity-Modulated Radiotherapies for Hepatocellular Carcinoma. Radiation Oncology, 9, 109. https://doi.org/10.1186/1748-717X-9-109

[6] da Costa, E.C., da Rosa, L.A.R. and Batista, D.V.S. (2015) Fetus Absorbed Dose Evaluation in Head and Neck Radiotherapy Procedures of Pregnant Patients. Applied Radiation and Isotopes, 100, 11-15. https://doi.org/10.1016/j.apradiso.2015.01.013

[7] van Westrhenen, A., Senders, J.T., Martin, E., DiRisio, A.C. and Broekman, M.L.D. (2018) Clinical Challenges of Glioma and Pregnancy: A Systematic Review. Journal of Neuro-Oncology, 139, 1-11. https://doi.org/10.1007/s11060-018-2851-3

[8] ICRP. (2007) The 2007 Recommendations of the International Commission on Radiological Protection. ICRP Publication 103. Annals of the ICRP, 37, 2-4. https://doi.org/10.1016/j.icrp.2007.10.003

[9] Kal, H.B. and Struikmans, H. (2005) Radiotherapy during Pregnancy: Fact and Fiction. Lancet Oncology, 6, 328-333. https://doi.org/10.1016/S1470-2045(05)70169-8

[10] Öğretici, A., Akbas, U., Köksal, C. and Bilge, H. (2016) Investigation of Conformal and Intensity Modulated Radiation Therapy Techniques to Determine the Absorbed Fetal Dose in Pregnant Patients with Breast Cancer. Medical Dosimetry, 41, 95-99. https://doi.org/10.1016/j.meddos.2015.10.001

[11] Sneed, P.K., Albright, N.W., Wara, W.M., Prados, M.D. and Wilson, C.B. (1995) Fetal Dose Estimates for Radiotherapy of Brain Tumors during Pregnancy. International Journal of Radiation Oncology, Biology, Physics, 32, 823-830. https://doi.org/10.1016/0360-3016(94)00456-U

[12] Mazonakis, M., Damilakis, J., Theoharopoulos, N., Varveris, H. and Gourtsoyiannis, N. (1999) Brain Radiotherapy during Pregnancy: An Analysis of Conceptus Dose Using Anthropomorphic Phantoms. The British Journal of Radiology, 72, 274-278. https://doi.org/10.1259/bjr.72.855.10396218

[13] Nuyttens, J.J., Prado, K.L., Jenrette, J.M. and Williams, T.E. (2002) Fetal Dose during Radiotherapy: Clinical Implementation and Review of the Literature. Cancer/ Radiothérapie, 6, 352-357. https://doi.org/10.1016/S1278-3218(02)00249-4

[14] Horowitz, D.P., et al. (2014) Fetal Radiation Monitoring and Dose Minimization during Intensity Modulated Radiation Therapy for Glioblastoma in Pregnancy. Journal of Neuro-Oncology, 120, 405-409. https://doi.org/10.1007/s11060-014-1565-4. 